\title{
X-RAY COMPUTED TOMOGRAPHY SIMULTANEOUS IMAGE RECONSTRUCTION AND CONTOUR DETECTION USING A HIERARCHICAL MARKOVIAN MODEL
}

\author{
Li WANG, Ali MOHAMMAD-DJAFARI, Nicolas GAC
}

\author{
Laboratoire des Signaux et Systeme, CentraleSupelec, Gif sur Yvette, France
}

\begin{abstract}
The 3D X-ray Computed Tomography (CT) is used in many domains. In medical imaging and industrial Non Destructive Testing (NDT) applications, this technique becomes of great interest. In these applications, very often, we need not only to reconstruct the image, but also to detect the contours between the homogeneous regions of the piecewise continuous image. Generally, contours are obtained by a post processing from the reconstructed image. In this paper, we propose a method to estimate image and contour simultaneously. For this we use the Bayesian approach with a prior model in which the relationship between the image and its contour is considered by using a hierarchical Markovian model, and use a sparsity enforcing prior model for the contours. This proposed method can be used for reconstructions when the image is piecewise continuous. The simulation results are compared with some state of the art methods, and they show the efficiency of simultaneously reconstructing and edge detecting by using proposed method.
\end{abstract}

Index Terms - Computed Tomography, Non Destructive Testing, Simultaneous reconstruction and contour detection, Markovian Model, and Generalized Student-t distribution

\section{INTRODUCTION}

$\mathrm{X}$-ray CT is used in many domains. The Radon transform is one of the commonly used model to describe it.

\subsection{X-ray Computed Tomography}

In the imaging systems, objects are often observed by using different techniques: X-rays [1], ultrasound, microwave, infra-red, etc. Each observation process provides different information on the object under study. In X-ray CT, the detected data provides information on the density distribution of the material inside the object.

The CT image plays an important role in medical and industrial Non Destructive Testing (NDT) applications. Its crosssectional images are used for diagnostic and therapeutic purposes in various medical disciplines. Industrial CT scanning has been used in many areas of industry for internal inspection of components. In the 3D CT problem, the Graphics Processing Unit (GPU), presented in details in [2], is always used to accelerate the computation.
In the work of Mumford and Shah in [3], they introduced the method which estimate the image and at the same time detect the segmentations and the contours. They estimate the variables by regularized optimization, in which the $l_{1}$ penalty is used to enforce sparsity. The inconvenient in their work is to chose the parameter values for all the regularization terms. In our work, we use a Bayesian method to estimate the image and the contours, estimating all the parameters at the same time. In this Bayesian method, a hierarchical Markovian prior model is defined, which combines the image and the contour informations. We use the heavy tailed distribution to enforce the sparsity of the contour. By using the contour information in the reconstruction of image, the edges of image are preserved. Comparing with the work of Mumford and Shah, our proposed method considers the relationships between (1) contour-projection gradient, (2) contour-image, (3) image-projection and (4) projection-projection gradient. The sparseness of contour is enforced and controlled by using a generalized Student-t distribution.

\subsection{Forward model: Radon Transform}

One of the most commonly used mathematical modelling of CT imaging is the Radon Transform (RT), presented with details in [4]. It can be written as:

$$
g(r, \phi)=\int_{L_{r, \phi}} f(x, y) \mathrm{d} l
$$

where $f(x, y)$ represents the image, $g$ the detected projection data, $r$ the perpendicular length from center point of coordinate and $\phi$ the considered $\mathrm{X}$ ray angle. $L_{r, \phi}$ is the length of ray $(r, \phi)$ passing through the image. One property that we consider in this work is:

$$
f(x, y) \rightarrow \mathrm{RT} \rightarrow g(r, \phi), \quad \ddot{f}(x, y) \rightarrow \mathrm{RT} \rightarrow \ddot{g}(r, \phi)
$$

where

$$
\ddot{f}(x, y)=\left(\frac{\partial^{2}}{\partial x^{2}}+\frac{\partial^{2}}{\partial y^{2}}\right) f(x, y), \quad \ddot{g}(r, \phi)=\frac{\partial^{2}}{\partial r^{2}} g(r, \phi)
$$

In the discretized form, the Radon Transform in Equation (1) can be written as:

$$
\boldsymbol{g}_{k}=\boldsymbol{H}_{k} \boldsymbol{f}+\boldsymbol{\epsilon}_{k} \quad \text { or } \quad \boldsymbol{g}=\boldsymbol{H} \boldsymbol{f}+\boldsymbol{\epsilon}
$$


where $\boldsymbol{g}_{k}$ is a vector representing the projection at angle $\phi_{k}$ and $\boldsymbol{g} \in \mathbb{R}^{M \times 1}$ represents all these vectors putting together. $\boldsymbol{H}_{k}$ and $\boldsymbol{H} \in \mathbb{R}^{M \times N}$ are their corresponding linear forward projection operators. Noted by $\ddot{f}$ the discretized $\ddot{f}(x, y)$ and by $\ddot{\boldsymbol{g}}_{k}$ discretized $\ddot{g}(r, \phi)$, Eq.(2) and Eq.(3) therefore are written as:

$$
\left\{\begin{array}{l}
\boldsymbol{g}=\boldsymbol{H} \boldsymbol{f}+\boldsymbol{\epsilon}, \ddot{\boldsymbol{g}}=\boldsymbol{H} \ddot{\boldsymbol{f}}+\ddot{\boldsymbol{\epsilon}} \\
\ddot{\boldsymbol{g}}=\boldsymbol{D}_{1} \boldsymbol{g}, \ddot{\boldsymbol{f}}=\boldsymbol{D}_{2} \boldsymbol{f}
\end{array}\right.
$$

where $\boldsymbol{D}_{1}$ represents the 1-dimensional second order derivation of $\boldsymbol{g}$ which can be implemented as the following convolution operation:

$$
\boldsymbol{D}_{1} \boldsymbol{g}=\left[\begin{array}{lll}
-1 & 2-1
\end{array}\right] * \boldsymbol{g},
$$

and $\boldsymbol{D}_{2}$ represents the discretized Laplacian operator which can be implemented by the following 2D convolution:

$$
\boldsymbol{D}_{2} \boldsymbol{f}=\left[\begin{array}{ccc}
0 & -1 & 0 \\
-1 & 4 & -1 \\
0 & -1 & 0
\end{array}\right] * \boldsymbol{f}
$$

The classical image reconstruction aims at estimating $f$ with $\boldsymbol{g}$. In this paper, we propose to use the forward relations in (5) to simultaneously reconstruct the image $f$ and detect the contours $\ddot{f}$ by using a Bayesian approach. For this, we use a hierarchical Markovian prior model to define the image $f$. Its hidden contour variables $q$, which is related to $\ddot{f}$, is used in this prior model. A sparsity enforcing Generalized Student- $t$ prior distribution is used to define the contours.

\section{PROPOSED METHOD OF RECONSTRUCTION OF OBJECT WHILE CONSIDERING CONTOURS}

\subsection{Forward model}

Starting from the forward model in (5) and assuming $\epsilon$ to be iid Gaussian distributed with variance $v_{\epsilon}$, we quickly get the likelihood:

$$
\left\{\begin{array}{l}
p\left(\boldsymbol{g} \mid \boldsymbol{f}, v_{\epsilon}\right)=\mathcal{N}\left(\boldsymbol{g} \mid \boldsymbol{H} \boldsymbol{f}, v_{\epsilon} \boldsymbol{I}\right) \\
p\left(\ddot{\boldsymbol{g}} \mid \ddot{\boldsymbol{f}}, v_{\epsilon}\right)=\mathcal{N}\left(\ddot{\boldsymbol{g}} \mid \boldsymbol{H} \ddot{\boldsymbol{f}}, 4 v_{\epsilon} \boldsymbol{I}\right) .
\end{array}\right.
$$

It is not difficult to demonstrate that the variance of $\ddot{\boldsymbol{\epsilon}}$, which is the second order derivation of $\boldsymbol{\epsilon}$, equals to $4 v_{\epsilon}$. The variance $v_{\epsilon}$ is supposed to be Inverse-Gamma distributed, so that the value is always positive and approaching to zero.

\subsection{Sparsity enforcing Student-t prior model for $\ddot{f}$}

When considering the piecewise-continuous image, its Laplacian is sparse with most of the part approaching to zero except the contours. As $\ddot{f}$ is sparse, we use a sparsity enforcing prior to define it. Typically three categories of such distributions are used to enforce sparseness: the Generalized Gaussian (GG) distributions, the Gaussian mixture distributions and the heavy-tailed distributions. The generalized Student- $t$ distribution is heavy-tailed, with the convenient that it can be expressed as the marginal of a Normal-Inverse Gamma distribution:

$$
\mathcal{S} t(x \mid \alpha, \beta)=\int \mathcal{N}(x \mid 0, z) \mathcal{I} \mathcal{G}(z \mid \alpha, \beta) \mathrm{d} z .
$$

With this property, we can set the following prior model for $\ddot{f}$ :

$$
\left\{\begin{array}{l}
p\left(\ddot{\boldsymbol{f}} \mid v_{b}\right)=\mathcal{N}\left(\ddot{\boldsymbol{f}} \mid 0, v_{b} \boldsymbol{I}\right) \\
p\left(v_{b} \mid \alpha, \beta\right)=\mathcal{I} \mathcal{G}\left(v_{b} \mid \alpha, \beta\right) .
\end{array}\right.
$$

where $\alpha, \beta$ are two hyper-parameters of generalized Student$\mathrm{t}$ distribution. With these two parameters, comparing with one-parametered standard Student-t distribution, the sparsity properties can be controlled more precisely.

\subsection{The image-contour hierarchical Markovian model}

By using Bayesian approach, prior informations are modeled as probabilistic distributions. Many prior models which are suitable for a Bayesian piecewise continuous CT image reconstruction method are discussed in the references [5-8]. In this paper we use a contour-depending Markovian prior model.

Let note by $f(r)$ the value of the pixel at the position $r$ and by $\boldsymbol{N}_{r}$ all the four neighbours pixels $r^{\prime}$. We use the following hierarchical contour-depending Markovian model to define $f$ :

$$
\begin{aligned}
& p\left(f(r) \mid \boldsymbol{f}_{-r}, \boldsymbol{q}, v_{a}\right) \\
& =\mathcal{N}\left(f(r) \mid \frac{1}{\sum_{r^{\prime} \in N_{r}}\left(1-q_{r^{\prime}}\right)} \sum_{r^{\prime} \in N_{r}}\left(1-q_{r^{\prime}}\right) f_{r^{\prime}}, v_{a}\right) \\
& \propto v_{a}^{-\frac{1}{2}} \exp \left\{-\frac{1}{2} v_{a}^{-1}\left(f(r)-\frac{\sum_{r^{\prime} \in N_{r}}\left(1-q_{r^{\prime}}\right) f_{r^{\prime}}}{\sum_{r^{\prime} \in N_{r}}\left(1-q_{r^{\prime}}\right)}\right)^{2}\right\}
\end{aligned}
$$

where $\boldsymbol{f}_{-r}$ represents all the elements in vector $\boldsymbol{f}$ except $f(r) . f_{r^{\prime}}$ represents the four neighbour pixels of $f_{r}$ and $v_{a}$ is the variance of the normal distribution. $q$ is the normalized $\ddot{\boldsymbol{f}}$, with the definition: $q=(|\ddot{\boldsymbol{f}}|-\min |\ddot{\boldsymbol{f}}|) /(\max |\ddot{\boldsymbol{f}}|-\min |\ddot{\boldsymbol{f}}|)$

This distribution describes the fact that: the influence of $f_{r^{\prime}}$ on $f_{r}$ depends on the gradient values at the positions $r^{\prime}$. For each neighbour pixel, when its gradient value is bigger, its influence on the current pixel is smaller. An example is given in Fig.(1). The pixel $f_{r}$ in this figure belongs to a Gaussian prior distribution: $p\left(f_{r} \mid \boldsymbol{f}_{r^{\prime}}, \boldsymbol{q}_{r^{\prime}}, v_{a}\right)=$ $\mathcal{N}\left(f_{r} \mid \frac{1}{2}\left(f_{r_{2}^{\prime}}+f_{r_{3}^{\prime}}\right), v_{a}\right)$.

\subsection{The model of the system}

With the previous propositions, we obtain a hierarchical model which reconstructs the image and contours simultaneously. $f$ belongs to a Gaussian prior distribution which depends on $\ddot{f}$, and $\ddot{f}$ belongs to a Generalized Student-t distribution which enforces the sparsity. 


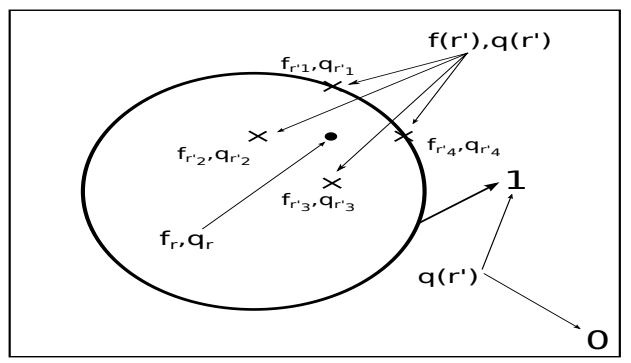

Fig. 1: Example of the contour-depending Markovian structured prior model.

By combining the proposed prior distributions of $f$, of $\ddot{f}$ and of all the other hyper parameters, we obtain the statistical distribution model of the system:

$$
\left\{\begin{array}{l}
p\left(\boldsymbol{g} \mid \boldsymbol{f}, v_{\epsilon}\right)=\mathcal{N}\left(\boldsymbol{g} \mid \boldsymbol{H} \boldsymbol{f}, v_{\epsilon} \boldsymbol{I}\right) \\
p\left(\ddot{\boldsymbol{g}} \mid \ddot{\boldsymbol{f}}, v_{\epsilon}\right)=\mathcal{N}\left(\ddot{\boldsymbol{g}} \mid \boldsymbol{H} \ddot{\boldsymbol{f}}, 4 v_{\epsilon} \boldsymbol{I}\right) \\
p\left(\boldsymbol{f} \mid \boldsymbol{q}, v_{a}\right)=\mathcal{N}\left(\boldsymbol{f} \mid \frac{1}{\sum_{r}\left(1-\boldsymbol{q}_{r}\right)} \sum_{r}\left(1-\boldsymbol{q}_{r}\right) \boldsymbol{f}_{r}, v_{a} \boldsymbol{I}\right) \\
p\left(\ddot{\boldsymbol{f}} \mid \boldsymbol{v}_{b}\right)=\mathcal{N}\left(\ddot{\boldsymbol{f}} \mid 0, \boldsymbol{\Sigma}_{b}\right) \text { where } \boldsymbol{\Sigma}_{b}=\operatorname{diag}\left[\boldsymbol{v}_{b}\right] \\
p\left(\boldsymbol{v}_{b} \mid \alpha_{b_{0}}, \beta_{b_{0}}\right)=\prod_{j} \mathcal{I} \mathcal{I}\left(v_{b_{j}} \mid \alpha_{b_{0}}, \beta_{b_{0}}\right) \\
p\left(v_{\epsilon} \mid \alpha_{\epsilon_{0}}, \beta_{\epsilon_{0}}\right)=\mathcal{I} \mathcal{G}\left(v_{\epsilon} \mid \alpha_{\epsilon_{0}}, \beta_{\epsilon_{0}}\right)
\end{array}\right.
$$

where $\boldsymbol{q}$ is defined below Eq.(9).

\subsection{The Bayes method}

The Bayesian inference [9] defines the relationship between prior and posterior distributions by:

$$
p(f, \theta \mid g)=\frac{p(g \mid f, \theta) p(f \mid \theta) p(\theta)}{p(g)} .
$$

By using the Bayes rule, with all the prior distributions in Equation (10), the posterior distribution is obtained:

$$
\begin{aligned}
p\left(\boldsymbol{f}, \ddot{\boldsymbol{f}}, v_{\epsilon}, \boldsymbol{v}_{b} \mid \boldsymbol{g}, \ddot{\boldsymbol{g}}\right) & \propto p\left(\boldsymbol{g} \mid \boldsymbol{f}, v_{\epsilon}\right) p\left(\ddot{\boldsymbol{g}} \mid \ddot{\boldsymbol{f}}, v_{\epsilon}\right) . \\
& p(\boldsymbol{f} \mid \boldsymbol{q}) p\left(\ddot{\boldsymbol{f}} \mid \boldsymbol{v}_{b}\right) p\left(\boldsymbol{v}_{b} \mid \alpha_{b_{0}}, \beta_{b_{0}}\right) p\left(v_{\epsilon} \mid \alpha_{\epsilon_{0}}, \beta_{\epsilon_{0}}\right)
\end{aligned}
$$

Here the conditional probabilities: $\boldsymbol{g}$ knowing $\boldsymbol{f}$ and $\ddot{g}$ knowing $\ddot{f}$, are independent.

\section{THE ESTIMATION OF VARIABLES}

A most commonly used estimation method in Bayesian approach is the Joint Maximum A Posterior (JMAP), presented in [10], which estimate the variables and parameters as the mode of the posterior distribution, by maximizing the posterior distribution:

$$
\left(\widehat{\boldsymbol{f}}, \widehat{\ddot{\boldsymbol{f}}}, \widehat{v}_{\epsilon}, \widehat{\boldsymbol{v}}_{b}\right)_{J M A P}=\arg \max _{\boldsymbol{f}, \ddot{\boldsymbol{f}}, v_{\epsilon}, \boldsymbol{v}_{b}}\left\{p\left(\boldsymbol{f}, \ddot{\boldsymbol{f}}, v_{\epsilon}, \boldsymbol{v}_{b} \mid \boldsymbol{g}, \ddot{\boldsymbol{g}}\right)\right\}
$$

\subsection{The iteration updating rules}

By maximizing the posterior distribution in Eq.(13), we get the updating equations. For $\widehat{f}$ and $\widehat{\ddot{f}}$, we use the descent gradient algorithm in order to avoid the calculation of the big size matrix inversion, $\left(\boldsymbol{H}^{t} \boldsymbol{H}\right)^{-1}$ :

$$
\begin{aligned}
& \text { iter }: \widehat{\boldsymbol{f}}^{(k+1)}=\widehat{\boldsymbol{f}}^{(k)}-\widehat{\gamma}_{1}^{(k)} \nabla \mathcal{J}\left(\widehat{\boldsymbol{f}}^{(k)}\right) \\
& \text { iter }: \widehat{\ddot{\boldsymbol{f}}}_{\ddot{\boldsymbol{g}}}^{(k+1)}=\widehat{\ddot{\boldsymbol{f}}}_{\ddot{\boldsymbol{g}}}^{(k)}-\widehat{\gamma}_{2}^{(k)} \nabla \mathcal{J}\left(\widehat{\ddot{\boldsymbol{f}}}_{\ddot{\boldsymbol{g}}}^{(k)}\right) \\
& \widehat{\ddot{\boldsymbol{f}}}=\left(\widehat{\ddot{\boldsymbol{f}}}_{\ddot{\boldsymbol{g}}}+\boldsymbol{D}_{2} \widehat{\boldsymbol{f}}\right) / 2 \\
& \widehat{v_{\epsilon}}=\frac{\beta_{\epsilon_{0}}+\frac{1}{2}\|\boldsymbol{g}-\boldsymbol{H} \widehat{\boldsymbol{f}}\|^{2}+\frac{1}{8}\|\ddot{\boldsymbol{g}}-\boldsymbol{H} \widehat{\ddot{\boldsymbol{f}}}\|^{2}}{\alpha_{\epsilon_{0}}+M+1} \\
& \widehat{v}_{b_{j}}=\frac{\beta_{b_{0}}+\frac{1}{2} \widehat{\ddot{f}}_{j}^{2}}{\alpha_{b_{0}}+\frac{3}{2}}
\end{aligned}
$$

where

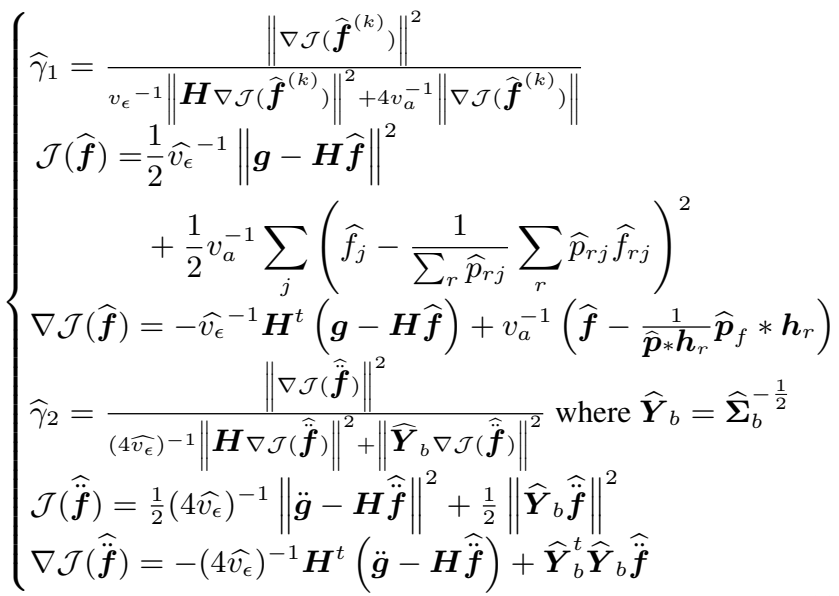

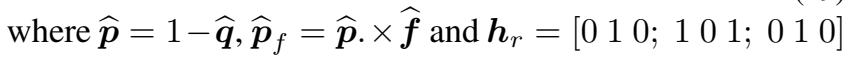
is a filter which calculates the sum of four neighbour pixels. Fig.(2) shows the directed acyclic graph (DAG) which presents the principal relationship of the updating of all the variables in the iterative updating rule in Eq.(14)-Eq.(19).

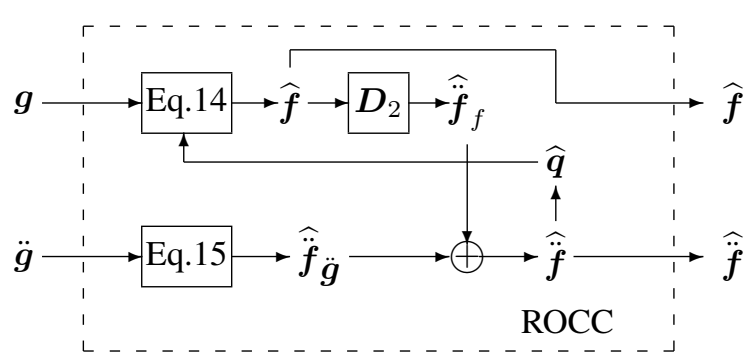

Fig. 2: DAG of proposed ROCC model. 


\section{SIMULATION RESULTS}

In the simulations, we use ROCC (Reconstruction of Object Considering Contours) to represent the proposed method. Two other hierarchical models are also used in the simulations: the model with $f$ belonging to a Gaussian distribution (GaussianF), and belonging to a Student-t distribution (StudentF).

Fig.(3) compares the performance of different methods applying on the Shepp Logan image of size $128 \times 128$. The Least Square(LS) method, Quadratic Regularization(QR) method, presented in [11,12], and the two hierarchical modelled methods are compared with the proposed ROCC method. The LS and $\mathrm{QR}$ methods performs poorly for this ill-posed inverse problem. The GaussianF method and StudentF method performs well when considering only the quality of the reconstructed image and the projections, but it can be seen that the ROCC method works better on the image reconstruction and at the same time gives more precise reconstructed contours, in which the contour is not used.

Fig.(4) shows the reconstructed Shepp Logan image and
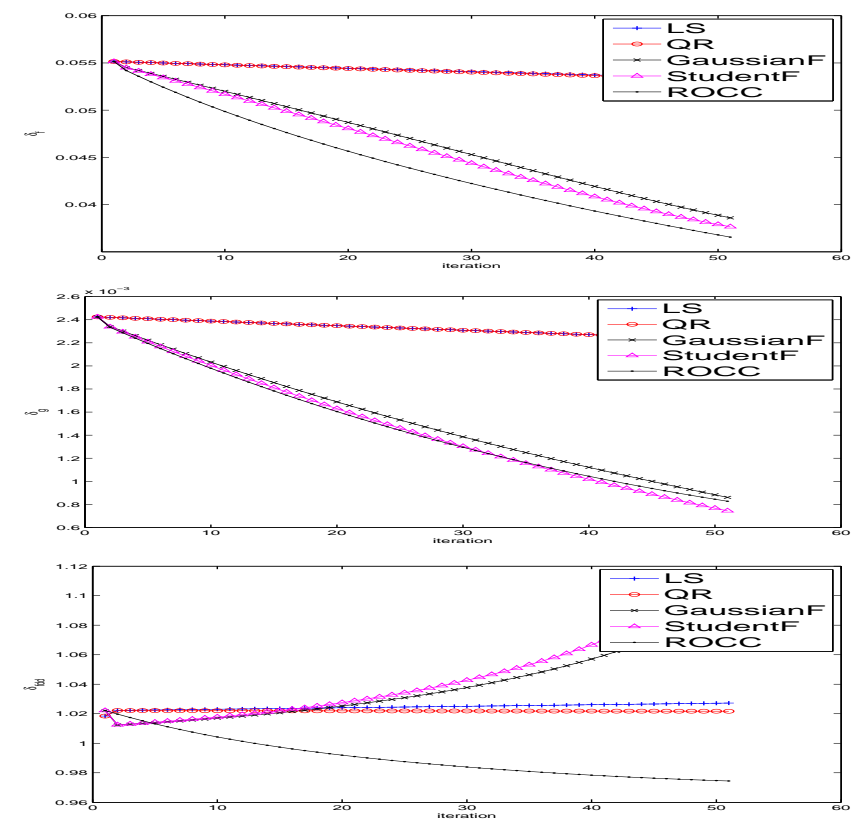

Fig. 3: Comparison of all the methods applying with the Shepp Logan image with 180 projections. These three figures, from top to bottom, show respectively the relative errors of the image $\| \widehat{\boldsymbol{f}}-$ $\boldsymbol{f}\left\|^{2} /\right\| \boldsymbol{f} \|^{2}$, the projections $\|\widehat{\boldsymbol{g}}-\boldsymbol{g}\|^{2} /\|\boldsymbol{g}\|^{2}$ and the contours $\| \widehat{\ddot{\boldsymbol{f}}}-$ $\ddot{\boldsymbol{f}}\left\|^{2} /\right\| \ddot{\boldsymbol{f}} \|^{2}$

its contours. We see from the reconstruction results that the small details of the image are retained, while the contours are obtained simultaneously.

The performance of different methods have also be compared by reconstructing the Walnut image of size $256 \times 256$. The comparison of the relative error of the reconstructed image

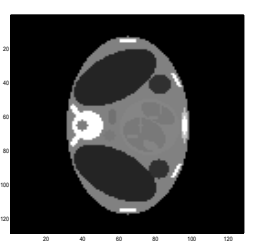

(a)

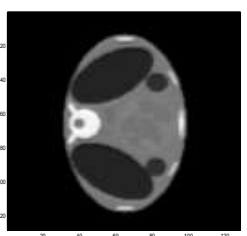

(b)

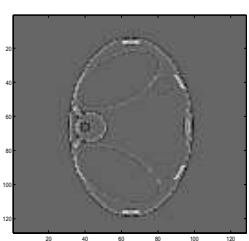

(c)
Fig. 4: The reconstruction results for the Shepp Logan image. Three images correspond to (a)the original image, (b)the reconstructed image and (c)the reconstructed contours.

is shown in Fig.(5), and the images are given in Fig.(6). The results show that the proposed ROCC method works better than the other methods.

Simulations have also be done with 90 projections, the pro-

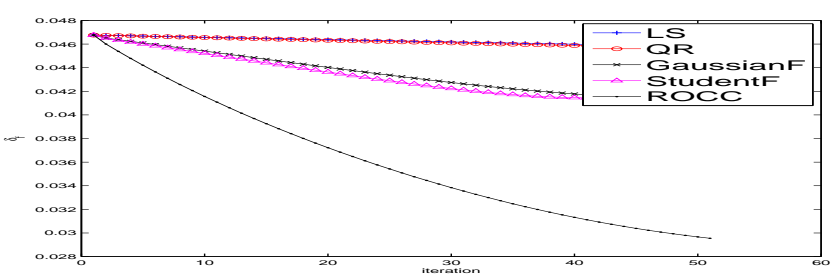

Fig. 5: Comparison of the relative error $\|\widehat{\boldsymbol{f}}-\boldsymbol{f}\|^{2} /\|\boldsymbol{f}\|^{2}$ of all the methods applying with the Walnut image with 180 projections.

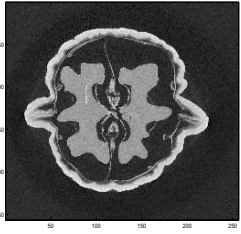

(a)

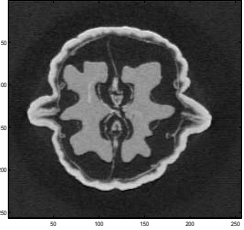

(b)

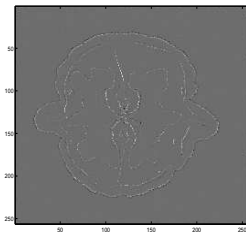

(c)
Fig. 6: The reconstructed results for the Walnut image. Three images correspond to (a)the original image, (b)the reconstructed image and (c)the reconstructed contours.

posed ROCC method always works better than the other state of the art methods.

\section{CONCLUSION}

In this paper we presented the $\mathrm{CT}$ image reconstruction by using a hierarchical Markovian model. This model preserves the edges of image, and reconstructs simultaneously the image and contours. Simulation results and comparisons with the state of the art methods shows that our proposed ROCC method performs better both on reconstruction of object and contours. This model can also be solved by using the Posterior Mean via Variational Bayesian Approach (VBA) method. Simulations in 3D application will also be done in our future work. 


\section{REFERENCES}

[1] Willi A Kalender, "X-ray Computed Tomography," Physics in Medicine and Biology, vol. 51, no. 13, pp. R29, 2006.

[2] Nicolas Gac, Alexandre Vabre, Ali Mohammad-Djafari, et al., "Multi GPU parallelization of 3D Bayesian CT algorithm and its application on real foam reonconstruction with incomplete data set," Proceedings FVR 2011, pp. 35-38, 2011.

[3] David Mumford and Jayant Shah, "Optimal approximations by piecewise smooth functions and associated variational problems," Communications on Pure and Applied Mathematics, vol. 42, no. 5, pp. 577-685, 1989.

[4] Stanley R Deans, The Radon Transform and some of its applications, Courier Corporation, 2007.

[5] Xiaojing $\mathrm{Gu}$, Henry Leung, and Xingsheng $\mathrm{Gu}$, "Bayesian sparse estimation using double lomax priors," Mathematical Problems in Engineering, vol. 2013, 2013.

[6] Michael Ting, Raviv Raich, and Alfred O Hero, "Sparse image reconstruction for molecular imaging," Image Processing, IEEE Transactions on, vol. 18, no. 6, pp. 1215-1227, 2009.

[7] Yang Chen, Jianhua Ma, Qianjin Feng, Limin Luo, Pengcheng Shi, and Wufan Chen, "Nonlocal prior Bayesian tomographic reconstruction," Journal of Mathematical Imaging and Vision, vol. 30, no. 2, pp. 133-146, 2008.

[8] Rafael Molina, Antonio López, José Manuel Martín, and Aggelos K Katsaggelos, "Variational posterior distribution approximation in bayesian emission tomography reconstruction using a Gamma mixture prior.," in VISAPP (Special Sessions), 2007, pp. 165-176.

[9] Mark E Glickman and David A van Dyk, "Basic bayesian methods," Topics in Biostatistics, pp. 319-338, 2007.

[10] Jinyi Qi and Richard M Leahy, "Resolution and noise properties of MAP reconstruction for fully 3-D PET," IEEE Transactions on Medical Imaging, vol. 19, no. 5, pp. 493-506, 2000.

[11] Michael Elad, Peyman Milanfar, and Ron Rubinstein, "Analysis versus synthesis in signal priors," Inverse Problems, vol. 23, no. 3, pp. 947, 2007.

[12] Simon Rit, David Sarrut, and Laurent Desbat, "Comparison of analytic and algebraic methods for motioncompensated cone-beam CT reconstruction of the thorax,” IEEE Transactions on Medical Imaging, vol. 28, no. 10, pp. 1513-1525, 2009. 